\title{
Trasplante hepático por hepatitis autoinmune y características asociadas con su recurrencia postrasplante: experiencia de un centro de trasplante hepático, Medellín, Colombia
}

\author{
Experience of a liver transplant center in Medellín, Colombia with liver \\ transplantation for autoimmune hepatitis and characteristics associated with \\ post-transplant recurrence
}

\author{
Gabriel Sebastián Díaz-Ramírez, ${ }^{1}$ Omar Yesid Martínez-Casas, ${ }^{1}$ Juan Ignacio Marín-Zuluaga, $, 2,3$ Jorge Hernando Donado-Gómez,,44 \\ Octavio Muñoz-Maya, ${ }^{2,3}$ Óscar Santos-Sánchez, ${ }^{2,3}$ Juan Carlos Restrepo-Gutiérrez., ${ }^{2,3}$
}

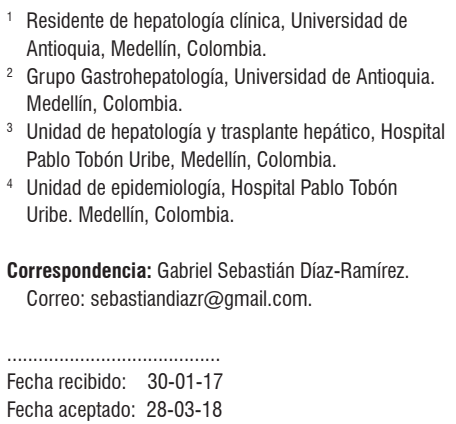

Fecha recibido: $30-01-17$

Fecha aceptado: 28-03-18

\begin{abstract}
Resumen
Introducción: la recurrencia de hepatitis autoinmune (HAl) postrasplante implica riesgo de cirrosis y pérdida del injerto. Se han propuesto factores de riesgo para su recurrencia, de los cuales pocos datos se conocen en pacientes latinoamericanos. Objetivos: describir las características de los pacientes con trasplante hepático por HAl y evaluar aquellas asociadas con su recurrencia durante el postrasplante. Métodos: cohorte histórica, incluyó pacientes con HAl diagnosticada a partir de los 16 años y que fueron llevados a trasplante hepático en un hospital universitario de Medellín, Colombia, entre enero de 2010 y septiembre de 2017. La recolección de la información se realizó a partir de los registros de historia clínica. Resultados: se incluyeron 25 pacientes. Se diagnosticó recurrencia en el 24\%. La mediana de seguimiento fue de 59,5 meses y de recurrencia 32,5 meses (rango 11-123 meses). El 100\% del grupo de recurrencia era de sexo femenino y ninguna de estas se trasplantó por insuficiencia hepática aguda. No hubo diferencias en las características pretrasplante y de tratamiento, aunque se encontró una mayor actividad inflamatoria bioquímica e histológica pretrasplante en el grupo de recurrencia. Del grupo con recurrencia, el 100\% recibió glucocorticoides a largo plazo y el 33,3\% tuvo pérdida del injerto relacionada con la recurrencia, por lo que requirieron retrasplante $(p=0,008)$. Conclusión: La recurrencia de HAI postrasplante hepático en nuestros pacientes es similar a la reportada mundialmente, es una causa de disfunción del injerto para tener en cuenta especialmente después del primer año postrasplante, predomina en mujeres. El 33,3\% de los pacientes requiere retrasplante hepático por disfunción del injerto.
\end{abstract}

Palabras clave

Hepatitis autoinmune, trasplante de hígado, Latinoamérica, Colombia.

\begin{abstract}
Introduction: The recurrence of post-transplant autoimmune hepatitis implies risk of cirrhosis and graft loss. Risk factors have been proposed for recurrence, of which few data are known in Latin American patients. Objectives: To describe the characteristics of patients with liver transplantation for autoimmune hepatitis and to evaluate those associated with their recurrence during post-transplant. Methods: Historical cohort included patients with autoimmune hepatitis diagnosed after the age of 16 years and who were taken to liver transplant in a university hospital in Medellin, Colombia between January 2010 and September 2017. Collection of information from the registers of clinical history. Results: 25 patients were included. Recurrence was diagnosed in $24 \%$. Median follow-up was 59.5 months and recurrence 32.5 months (range $11-123$ months). 100\% of the recurrence group were women and none of these were transplanted due to acute liver failure. There were no differences in the pretransplant and treatment characteristics, although a higher biochemical and histological inflammatory activity was found pre-transplant in the recurrence group. Of the group with recurrence, 100\% received long-term glucocorticoids and $33.3 \%$ had graft loss related to recurrence requiring retransplantation $(p=0.008)$. Conclusion: The recurrence of autoimmune hepatitis after liver transplantation in our patients is similar to that reported worldwide, is a cause of graft dysfunction to be taken into account especially after the first year post-transplant, it predominates in women. $33.3 \%$ of patients require hepatic retransplantation due to graft dysfunction.
\end{abstract}

Keywords

Autoimmune hepatitis, liver transplant, Latin America, Colombia. 


\section{INTRODUCCIÓN}

Desde su primera descripción, en 1950, la hepatitis autoinmune (HAI) se ha considerado una enfermedad hepática inflamatoria crónica (1), hasta el momento de etiología desconocida y causante de insuficiencia hepática aguda, de cirrosis hepática y una de las indicaciones de trasplante hepático (2).

La HAI se ha descrito como indicación de trasplante hepático hasta en el 5\% de las series a nivel mundial (3). En Colombia, las enfermedades hepáticas autoinmunes son la causa del $12 \%$ de los trasplantes hepáticos en adultos (4). La supervivencia postrasplante hepático de los pacientes trasplantados por HAI es buena, alcanzando el $90 \%$ y $80 \%$ a 1 y 5 años, respectivamente (5); sin embargo, se describe una recurrencia de HAI entre el $12 \%$ y $46 \%(6,7,8)$ que puede llevar a cirrosis y pérdida del injerto hasta el en 50\% de los casos $(3,9)$, con requerimiento de retrasplante, y existe riesgo de muerte.

Los factores de riesgo asociados con la recurrencia de HAI aún no están claramente dilucidados; sin embargo, se describen algunos como la suspensión temprana de glucocorticoides en el período postrasplante, la incompatibilidad del sistema del antígeno leucocitario humano (HLA) entre el donante y el receptor, los esquemas de inmunosupresión postrasplante y el grado de actividad inflamatoria (bioquímica e histológica) al momento del trasplante $(8,10)$.

El objetivo de este estudio es describir las características de los pacientes con trasplante hepático por HAI, evaluar aquellas asociadas con recurrencia de HAI en el postrasplante y describir el curso postrasplante de los pacientes trasplantados por HAI en un hospital de referencia para trasplante hepático en Colombia.

\section{MATERIALES Y MÉTODOS}

\section{Población}

Estudio observacional de cohorte histórica que incluyó pacientes con diagnóstico de HAI acorde con los criterios simplificados para el diagnóstico de HAI del Grupo Internacional de Hepatitis Autoinmune (GIHA) (11) a partir de los 16 años de edad y que fueron llevados a trasplante hepático en el Hospital Pablo Tobón Uribe de Medellín, Colombia, entre el 1 de enero de 2010 y el 30 de septiembre de 2017.

Se excluyeron pacientes con ausencia de datos clínicos, bioquímicos e histológicos completos, y aquellos con trasplante hepático por síndromes de sobreposición de hepatitis autoinmune-colangitis biliar primaria (HAI-CBP) o hepatitis autoinmune-colangitis esclerosante primaria (HAI-CEP), u otras enfermedades hepáticas crónicas.

\section{Variables}

La recolección de los datos se realizó mediante la revisión de historias clínicas del registro electrónico del hospital con un instrumento de recolección previamente diseñado.

Se recogieron variables demográficas, clínicas, quirúrgicas, serológicas, radiológicas, histológicas y de tratamiento durante los períodos pretrasplante y postrasplante hepático.

\section{Recurrencia de HAI}

La recurrencia de HAI se definió por los hallazgos de la histología hepática (infiltrados linfoplasmocíticos portales y periportales), bioquímico: elevación de transaminasas al menos 2 veces por encima del límite superior de normalidad; y la ausencia de otras causas de disfunción del injerto hepático.

\section{Análisis estadístico}

Se realizó un análisis descriptivo de las variables en el que las categóricas se presentan como frecuencias absolutas y relativas; las variables continuas como media y desviación estándar si siguen una distribución normal o mediana, y rango intercuartílico (RIC) si no siguen una distribución normal según la prueba de Kolmogorov Smirnov. Las variables cualitativas se compararon con la prueba exacta de Fisher y las continuas con la prueba $t$ de Student o U de Mann Whitney, según su distribución. Se realizó un seguimiento de los pacientes hasta el 30 de septiembre de 2017 o hasta la última fecha que se tenía documentada en la historia clínica a partir de la cual se consideró como dato censurado si no había presentado ninguno de los desenlaces medidos. Se empleó el paquete estadístico SPSS versión 20 registrada de la Universidad de Antioquia.

El manuscrito final se adhirió a las recomendaciones STROBE para el reporte de estudios observacionales.

\section{Aspectos éticos}

El estudio se adhirió a los lineamientos de la declaración de Helsinki versión 2013 para la investigación en seres humanos y a la resolución 008430 de 1993 sobre investigación clínica en Colombia, y fue aprobado por el comité de ética del hospital.

\section{RESULTADOS}

Un total de 25 pacientes con HAI se trasplantaron entre el 1 de enero de 2010 y el 30 de septiembre de 2017; de estos, se diagnosticó recurrencia de HAI en 6 (24\%) durante el postrasplante. La tasa de incidencia de recurrencia fue de 0,59 casos por 100 personas/año. La mediana de seguimiento fue 
59,5 meses (RIC: 19,2-94). La comorbilidad más frecuente fue el hipotiroidismo (7 pacientes, 22,5\%). Las características de los pacientes al momento del diagnóstico de HAI y previo al trasplante hepático se describen en la Tabla 1.

La principal indicación de trasplante en ambos grupos fue la cirrosis hepática y sus complicaciones. El 16\% de los pacientes recibió el trasplante por insuficiencia hepática aguda (todas de tipo subagudo), ninguno de estos tuvo recurrencia postrasplante de HAI $(p=0,218)$.

El 68\% de todos los pacientes tuvo al menos un autoanticuerpo positivo. Los anticuerpos antinucleares (ANA) fueron positivos en el $68 \%$ de los pacientes y los anticuerpos antimúsculo liso (AsMA) en el 32\%. No hubo diferencias en cuanto al perfil de autoanticuerpos entre los 2 grupos. El grupo de recurrencia de HAI tuvo mayores niveles de IgG sérica pretrasplante sin alcanzar significancia estadística $(\mathrm{p}=0,840)$.

La mayoría de los pacientes en ambos grupos recibió tratamiento farmacológico para HAI en el pretrasplante, y el tratamiento más frecuentemente utilizado fue la combinación de glucocorticoides e inmunomodulador (azatioprina $[\mathrm{AZA}]$ o micofenolato de mofetilo $[\mathrm{MMF}])$. En el grupo que no tuvo recurrencia, 6 pacientes $(31,6 \%)$ no recibieron tratamiento farmacológico pretrasplante; de estos, en $4(66,6 \%)$ la indicación de trasplante fue insuficiencia hepática aguda y 2 restantes tuvieron cirrosis sin actividad inflamatoria reflejada en la bioquímica hepática.

Hubo una mayor frecuencia de recaídas de HAI en el grupo de recurrencia postrasplante $(33,3 \%$ frente a $15,8 \%$; $\mathrm{p}=0,347$ ), la principal causa de recaída fue la adherencia irregular al tratamiento farmacológico.

En la Tabla 2 se describen las características de los pacientes al momento del trasplante hepático y en el postrasplante. La mediana de edad al momento del trasplante hepático y el tiempo entre el diagnóstico de HAI y el trasplante fue menor en el grupo que presentó recurrencia postrasplante.

La mediana de recurrencia postrasplante de HAI fue de 32,5 meses (RIC: 17,7-72) (Figura 1).

No se encontró diferencia significativa en cuanto a la positividad de aloanticuerpos (anticuerpos de donante específico) entre los 2 grupos.

$\mathrm{Al}$ comparar los 2 grupos en cuanto a los hallazgos histológicos del hígado explantado, se encontró una mayor

Tabla 1. Características de los pacientes con HAI durante el período pretrasplante hepático

\begin{tabular}{|c|c|c|c|c|}
\hline Características & $\begin{array}{l}\text { Todos los pacientes } \\
\quad(n=25)\end{array}$ & $\begin{array}{c}\text { Sin recurrencia } \\
\text { postrasplante }(n=19)\end{array}$ & $\begin{array}{c}\text { Recurrencia } \\
\text { postrasplante }(n=6)\end{array}$ & $\begin{array}{l}\text { Valor } \\
\text { de } p\end{array}$ \\
\hline Sexo femenino (\%) & $19(76)$ & $13(68,4)$ & $6(100)$ & 0,114 \\
\hline Edad en años al diagnóstico de HAl-mediana (RIC) & $43(24,2-72,2)$ & $43(28,5-57)$ & $41(18-61)$ & 0,875 \\
\hline Antecedente de enfermedad autoinmune (\%) & $1(4)$ & $0(0)$ & $1(16,7)$ & 0,06 \\
\hline \multicolumn{5}{|l|}{ Indicación del trasplante hepático } \\
\hline Insuficiencia hepática aguda (\%) & $4(16)$ & $4(21)$ & $0(0)$ & 0,218 \\
\hline Cirrosis hepática (\%) & $21(84)$ & $15(79)$ & $6(100)$ & 0,218 \\
\hline Child-Pugh pretrasplante-mediana (RIC) & $10(8-12)$ & $10(8-12)$ & $9(7-12)$ & 0,740 \\
\hline MELD pretrasplante-mediana (RIC) & $16(12-22)$ & $15,5(11-25)$ & $17,5(14-20)$ & 0,925 \\
\hline \multicolumn{5}{|l|}{ Complicaciones de la cirrosis } \\
\hline Ascitis (\%) & $17(68)$ & $13(68,4)$ & $4(66,6)$ & 0,936 \\
\hline Encefalopatía (\%) & $9(36)$ & $7(36,8)$ & $2(33,3)$ & 0,872 \\
\hline Várices esofágicas (\%) & $8(32)$ & $6(31,5)$ & $2(33,3)$ & 0,936 \\
\hline Hepatocarcinoma (\%) & $1(4)$ & $0(0)$ & $1(16,6)$ & 0,068 \\
\hline Anticuerpos antinucleares $\geq 1: 40(\%)$ & $17(68)$ & $13(68,4)$ & $4(66)$ & 0,936 \\
\hline Anticuerpos antimúsculo liso positivos (\%) & $8(32)$ & $6(31,5)$ & $3(50)$ & 0,412 \\
\hline IgG sérica mg/dL mediana (RIC) & $2224(1621-2641)$ & $2174(1592-2919)$ & $2349(1600-2350)$ & 0,840 \\
\hline \multicolumn{5}{|l|}{ Tratamiento antes del trasplante } \\
\hline Ninguno (\%) & $7(28)$ & $6(31,6)$ & $1(16,7)$ & 0,477 \\
\hline Esteroide (\%) & $2(8)$ & $1(5,2)$ & $1(16,7)$ & 0,368 \\
\hline Esteroide + inmunomodulador (\%) & $13(52)$ & $9(47,4)$ & $4(66,6)$ & 0,412 \\
\hline Recaídas (\%) & $5(20)$ & $3(15,8)$ & $2(33,3)$ & 0,347 \\
\hline Mediana de seguimiento en meses (RIC) & $59,5(19,2-94)$ & $43(17-74,5)$ & $97(40-152)$ & 0,170 \\
\hline
\end{tabular}

IgG: inmunoglobulina G; MELD: model for end-stage liver disease. 
Tabla 2. Características de los pacientes con HAI durante el momento del trasplante hepático y período postrasplante

\begin{tabular}{|c|c|c|c|c|}
\hline Características & $\begin{array}{l}\text { Todos los pacientes } \\
\quad(n=25)\end{array}$ & $\begin{array}{c}\text { Sin recurrencia } \\
\text { postrasplante }(n=19)\end{array}$ & $\begin{array}{c}\text { Recurrencia } \\
\text { postrasplante }(n=6)\end{array}$ & $\begin{array}{l}\text { Valor } \\
\text { de } p\end{array}$ \\
\hline Edad en años al momento del trasplante-mediana (RIC) & $42,5(28-58,7)$ & $43(30-59,5)$ & $38(21-60)$ & 0,664 \\
\hline $\begin{array}{l}\text { Semanas entre el diagnóstico de HAl y el trasplante- } \\
\text { mediana (RIC) }\end{array}$ & $24,4(9-172,3)$ & $28,3(2,3-179,9)$ & $18,5(15,8-129,6)$ & 0,869 \\
\hline AST U/L pretrasplante-mediana (RIC) & $103(70,5-178)$ & $103(70-323)$ & $118,5(82,7-178)$ & 0,815 \\
\hline ALT U/L pretrasplante-mediana (RIC) & $101(61-149,5)$ & $101(53-177)$ & $95,5(61,2-141,2)$ & 0,969 \\
\hline FA U/L pretrasplante-mediana (RIC) & $198(130-311,5)$ & $167(125-313)$ & $237,5(169,2-346,2)$ & 0,586 \\
\hline Bilirrubina mg/dL pretrasplante-mediana $(\mathrm{RIC})$ & $6,1(3,7-21)$ & $7,4(5,2-30)$ & $4,2(1,6-6,25)$ & 0,056 \\
\hline Positividad de aloanticuerpos (\%) & $2(8)$ & $2(10,5)$ & $0(0)$ & 0,406 \\
\hline Actividad inflamatoria moderada a severa en el explante (\%) & $19(76)$ & $13(68,4)$ & $6(100)$ & 0,114 \\
\hline TIF en minutos-mediana (RIC) & $380(280-400)$ & $380(317,5-420)$ & $300(265-385)$ & 0,228 \\
\hline TIC en minutos-mediana (RIC) & $29(25-32)$ & $30,5(25,7-36,2)$ & $26(24,5-29)$ & 0,151 \\
\hline Basiliximab (\%) & $1(4)$ & $1(5,3)$ & $0(0)$ & 0,568 \\
\hline \multicolumn{5}{|l|}{ Inmunosupresión postrasplante } \\
\hline PRED (\%) & $24(96)$ & $18(94,4)$ & $6(100)$ & 0,568 \\
\hline PRED hasta el diagnóstico de la recurrencia (\%) & $23(92)$ & $17(89,4)$ & $6(100)$ & 0,406 \\
\hline \multicolumn{5}{|l|}{ Inhibidor de calcineurina } \\
\hline $\mathrm{CIC}(\%)$ & $10(40)$ & $6(31,6)$ & $4(66,7)$ & 0,126 \\
\hline Tacrolimus (\%) & $15(60)$ & $13(68,4)$ & $2(33,3)$ & 0,126 \\
\hline \multicolumn{5}{|l|}{ Anti metabolito } \\
\hline AZA $(\%)$ & $8(32)$ & $6(31,6)$ & $2(33,3)$ & 0,936 \\
\hline MMF (\%) & $17(68)$ & $13(68,4)$ & $4(66,7)$ & 0,936 \\
\hline \multicolumn{5}{|l|}{ Complicaciones postrasplante } \\
\hline Rechazo celular agudo moderado a severo (\%) & $11(44)$ & $8(42,1)$ & $3(50)$ & 0,727 \\
\hline Infección por CMV & $2(8)$ & $2(10,6)$ & $0(0)$ & 0,406 \\
\hline $\begin{array}{l}\text { Meses entre el trasplante y la recurrencia de } \\
\text { HAl-mediana (RIC) }\end{array}$ & & & $32,5(17,7-72)$ & \\
\hline Retrasplante hepático (\%) & $2(8)$ & $0(0)$ & $2(33,3)$ & 0,008 \\
\hline Muerte (\%) & $3(12)$ & $3(15,8)$ & $0(0)$ & 0,298 \\
\hline
\end{tabular}

CIC: ciclosporina; CMV: citomegalovirus; PRED: prednisolona; TIC: tiempo de isquemia caliente; TIF: tiempo de isquemia fría.

actividad inflamatoria en los pacientes que tuvieron recurrencia postrasplante $(\mathrm{p}=0,114)$.

Más del $90 \%$ de los pacientes recibieron tratamiento con prednisolona durante el período postrasplante, el cual se mantuvo a largo plazo. Al momento del diagnóstico de la recurrencia de HAI, el 100\% de los pacientes continuaban recibiendo prednisolona.

El esquema de inmunosupresión postrasplante más frecuentemente utilizado fue la combinación de antimetabolito (MMF o AZA) e inhibidor de calcineurina (tacrolimus o CIC). En ambos grupos, el antimetabolito más prescrito fue el MMF. En cuanto al inhibidor de calcineurina, el grupo de recurrencia postrasplante tuvo un mayor uso de
CIC y el de no recurrencia de tacrolimus, diferencias no significativas estadísticamente.

Dos pacientes $(33,3 \%)$ del grupo de recurrencia de HAI requirieron retrasplante $(p=0,008)$ debido a disfunción del injerto. La tasa de incidencia de retrasplante fue de 0,78 casos por 100 personas/año. Ambos pacientes fueron del sexo femenino, tenían actividad inflamatoria moderada en el explante e historia de rechazo celular agudo. El tiempo entre la recurrencia de HAI y el retrasplante fue de 30 y 113 meses.

En total murieron 3 pacientes (todos del grupo sin recurrencia de HAI) por complicaciones infecciosas, 2 por choque séptico (a los 7 y 17 meses postrasplante) y 1 a los 61 meses por tuberculosis pulmonar e infección por CMV. 


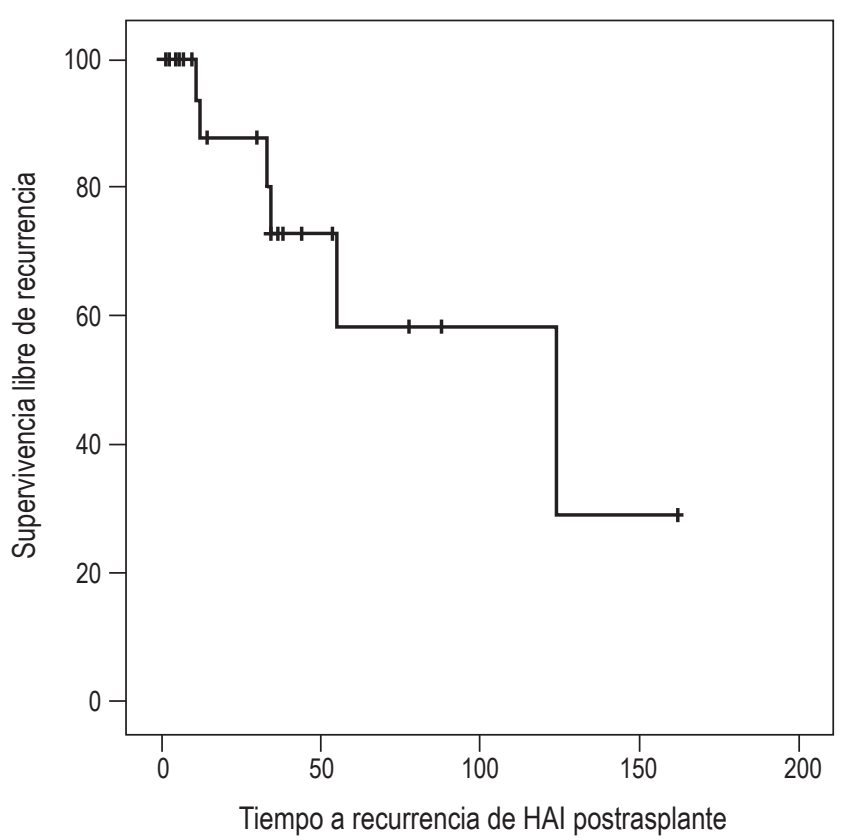

Figura 1. Supervivencia (en meses) libre de recurrencia de hepatitis autoinmune durante el período postrasplante hepático.

Las características individuales de los pacientes con recurrencia postrasplante de HAI se describen en la Tabla 3.

\section{DISCUSIÓN}

La HAI es una causa importante de trasplante hepático a nivel mundial (2); sin embargo, la mayoría de datos proviene de estudios en población caucásica ya que poco se conoce del curso de la enfermedad en pacientes latinoame- ricanos (12). En estudios recientemente publicados por nuestro grupo, se encontró que las enfermedades hepáticas autoinmunes son causa del $12 \%$ de los trasplantes hepáticos en adultos colombianos (4) y que el 10,1\% de los pacientes con HAI (incluidos los síndromes de sobreposición HAICBP y HAI-CEP) requirió trasplante hepático durante el curso de la enfermedad con una incidencia de 2,5 trasplantes por 100 pacientes/año (intervalo de confianza [IC] 95\%: 1,7-2,7) (12). Se encontró recurrencia de HAI en el $24 \%$ de los pacientes trasplantados y una tasa de incidencia de recurrencia de 0,59 casos por 100 personas/año, similar a lo reportado en la literatura mundial $(6,7,8)$; y aunque el tamaño de muestra es pequeño y limitó encontrar diferencias significativas entre los grupos, en la mayoría de las variables evaluadas hay varios aspectos que se considera importante resaltar a continuación.

La mediana de recurrencia fue de 32,5 meses (rango 11-123 meses) y a nivel mundial se describe una mediana de 26,4 (13). Esto sugiere que la recurrencia de HAI es una entidad por considerar cuando se presenta disfunción del injerto, especialmente después del primer año postrasplante.

Las características pretrasplante asociadas con recurrencia son variables, al igual que lo reportado por MontanoLoza en pacientes canadienses (8), ninguno de los pacientes trasplantados por insuficiencia hepática aguda tuvo recurrencia de HAI y, aunque no se ha encontrado asociación estadística, esta podría estar limitada por el escaso número de pacientes incluidos en los estudios. En ese mismo estudio, el grado de inflamación hepática (reflejado por marcadores serológicos como la IgG o por histología) previa al trasplante estuvo asociado significativamente en el análisis multivariado con recurrencia de HAI con Hazard ratio (HR): 6,9 y 7,5 para la inflamación moderada a severa

Tabla 3. Características de los pacientes con recurrencia de HAI postrasplante

\begin{tabular}{|c|c|c|c|c|c|c|c|c|c|c|}
\hline Paciente & 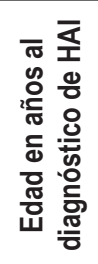 & 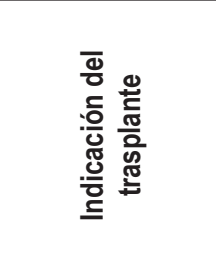 & 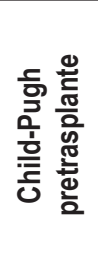 & 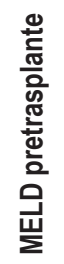 & 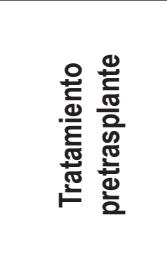 & 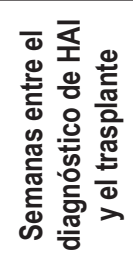 & 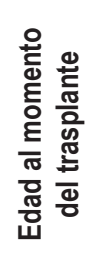 & 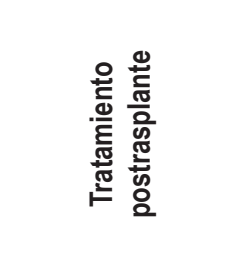 & 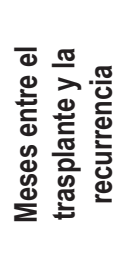 & 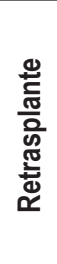 \\
\hline 1 & 66 & Cirrosis hepática & 12 & 19 & AZA, PRED & 16 & 66 & PRED, AZA, CIC & 55 & No \\
\hline 2 & 57 & Cirrosis hepática & 7 & 22 & Ninguno & 19 & 58 & PRED, MMF, TAC & 11 & No \\
\hline 3 & 41 & Cirrosis hepática & 11 & 16 & Ninguno & 16 & 42 & PRED, MMF, CIC & 33 & Sí \\
\hline 4 & 19 & Cirrosis hepática & 8 & 12 & CIC, PRED & 217 & 23 & PRED, MMF, TAC & 20 & No \\
\hline 5 & SD & Cirrosis hepática & 8 & 15 & CIC, PRED & SD & 34 & PRED, AZA, CIC & 123 & No \\
\hline 6 & 17 & Cirrosis hepática & 12 & 20 & PRED, MMF & 42 & 17 & PRED, MMF, CIC & 32 & Sí \\
\hline
\end{tabular}

SD: sin dato. 
a nivel histológico y niveles de $\operatorname{Ig} \mathrm{G}$ sérica, respectivamente; esto sugiere alcanzar una remisión bioquímica e histológica pretrasplante podría reducir la recurrencia de HAI. En el grupo de recurrencia de HAI, se encontró mayores niveles de IgG sérica y mayor actividad histológica inflamatoria (100\% frente a $68,4 \%$ ), ambos sin significancia estadística posiblemente debido al pequeño número de pacientes.

$\mathrm{Al}$ evaluar las características postrasplante no se encontró asociación entre los diferentes esquemas de tratamiento inmunosupresor postrasplante y la recurrencia de HAI, al igual que lo descrito en las revisiones sistemáticas a nivel mundial $(13,14)$; a diferencia de otras patologías como la colangitis biliar primaria (CBP), en la que se ha descrito mayor recurrencia postrasplante con el uso de tacrolimus frente a CIC (14). El uso indefinido de glucocorticoides a dosis bajas en el postrasplante ha demostrado disminuir la recurrencia de HAI en estudios previos: $0 \%$ y $11 \%$ a 1 y 10 años, respectivamente (15). Sin embargo, en nuestro estudio, a pesar de que el $92 \%$ del total de pacientes trasplantados por HAI y el 100\% del grupo de recurrencia recibieron prednisolona a dosis bajas a largo plazo, hubo una recurrencia del $24 \%$. No se pudo realizar análisis comparativo en cuanto a la recurrencia de HAI con el uso o no de prednisolona a largo plazo debido a que la administración de glucocorticoides indefinidamente es una conducta que se ha instaurado en nuestro grupo desde hace algunos años en estos pacientes.

La recurrencia de HAI postrasplante es una causa importante de disfunción del injerto, de cirrosis y pérdida del mismo, con requerimiento de retrasplante hepático (9) y de muerte; debido a esto, es importante identificar los pacientes con riesgo de recurrencia y establecer acciones para disminuir dicho riesgo, tanto en el período pretrasplante (búsqueda de remisión bioquímica e histológica) como en el postrasplante (la administración de dosis bajas de glucocorticoides a largo plazo) (14), y la realización de biopsia hepática de seguimiento ya que se ha demostrado que la recurrencia histológica precede a la recurrencia clínica y bioquímica (16). Se encontró significancia estadística en cuanto al requerimiento de retrasplante hepático $(33,3 \%$ frente a $0 \%, p=0,008)$, que fue mayor en el grupo de recurrencia. El retrasplante hepático, aunque es una opción terapéutica descrita hasta en el $50 \%$ de los casos de los pacientes con recurrencia (9), está asociada con mayores costos, mayor morbilidad y no está siempre disponible, especialmente en regiones con bajas tasas de donación de órganos.

En cuanto a las limitaciones, se trata de un estudio de un solo centro; las limitaciones inherentes a un estudio retrospectivo, especialmente al sesgo de información ya que los datos se recogieron a partir del registro de historias clínicas del hospital; además, el tamaño de muestra fue pequeño, lo que no permitió hacer inferencias con significancia estadística; otra limitación fue que no se evaluaron los HLA en los pacientes, debido a que se ha encontrado asociación de los mismos con recurrencia y peores desenlaces en el postrasplante, especialmente en pacientes con HLA-DR3 $(17,18)$. Además, no existen criterios validados en estudios para diagnóstico de recurrencia de HAI y los criterios simplificados para el diagnóstico pretrasplante (11) no se recomiendan en el postrasplante; sin embargo, los hallazgos histológicos (infiltrados linfoplasmocíticos portales y periportales), la elevación de transaminasas y de $\operatorname{IgG}$ sérica, y la ausencia de otras etiologías ayudan a confirmar el diagnóstico de recurrencia $(14,15)$; nuestros pacientes cumplieron con estos criterios. Por último, no se realizaron biopsias de seguimiento por protocolo en ausencia de alteración del perfil bioquímico hepático, por lo que se puede subestimar la incidencia de recurrencia ya que, aunque es infrecuente, se ha descrito recurrencia de HAI en pacientes sin elevación de transaminasas (19).

Se destacan como fortalezas que se trata de un estudio realizado en un centro de referencia para enfermedades hepáticas y trasplante hepático a nivel nacional que cuenta con el número más grande de pacientes con HAI reportado en Colombia y Latinoamérica (12), y el seguimiento en el tiempo (mediana: 59,5 meses) permitió conocer el comportamiento de la HAI en la población adulta tanto en el pretrasplante como en el postrasplante.

\section{CONCLUSIONES}

La HAI es una importante indicación de trasplante hepático a nivel mundial y en Colombia. Su recurrencia en el postrasplante en nuestra población es similar a la reportada a nivel mundial. Es una causa de disfunción del injerto para tener en cuenta especialmente después del primer año postrasplante, ya que el $33,3 \%$ de los pacientes con recurrencia requieren retrasplante. Un estudio multicéntrico de los centros de trasplante en Colombia con un mayor número de pacientes ayudaría a determinar los factores asociados con recurrencia de HAI postrasplante en la población colombiana.

\section{Fuentes de financiación}

Ninguna.

\section{Conflicto de interés}

Los autores no manifiestan conflicto de interés. 


\section{REFERENCIAS}

1. Heneghan MA, Yeoman $\mathrm{AD}$, Verma S, Smith $\mathrm{AD}$, Longhi MS. Autoimmune hepatitis. Lancet. 2013;382(9902):143344. doi: 10.1016/S0140-6736(12)62163-1.

2. European Association for the Study of the Liver. EASL Clinical Practice Guidelines: Autoimmune hepatitis. J Hepatol. 2015;63(4):971-1004. doi: 10.1016/j. jhep.2015.06.030.

3. Reich DJ, Fiel I, Guarrera JV, Emre S, Guy SR, Schwartz $\mathrm{ME}$, et al. Liver transplantation for autoimmune hepatitis. Hepatology. 2000;32(4 Pt 1):693-700. doi: 10.1053/ jhep.2000.16666.

4. Santos O, Londoño M, Marín J, Muñoz O, Mena Á, Guzmán C, et al. An experience of liver transplantation in Latin America: a medical center in Colombia. Colomb Med (Cali). 2015;46(1):8-13.

5. Mottershead M, Neuberger J. Transplantation in autoimmune liver diseases. World J Gastroenterol. 2008;14(21):3388-95. doi: 10.3748/wjg.14.3388.

6. Liberal R, Zen Y, Mieli-Vergani G, Vergani D. Liver transplantation and autoimmune liver diseases. Liver Transpl. 2013;19(10):1065-77. doi: 10.1002/lt.23704.

7. Czaja AJ. Diagnosis and Management of Autoimmune Hepatitis: Current Status and Future Directions. Gut Liver. 2016;10(2):177-203. doi: 10.5009/gnl15352.

8. Montano-Loza AJ, Mason AL, Ma M, Bastiampillai RJ, Bain VG, Tandon P. Risk factors for recurrence of autoimmune hepatitis after liver transplantation. Liver Transpl. 2009;15(10):1254-61. doi: 10.1002/lt.21796.

9. Ratziu V, Samuel D, Sebagh M, Farges O, Saliba F, Ichai P, et al. Long-term follow-up after liver transplantation for autoimmune hepatitis: evidence of recurrence of primary disease. J Hepatol. 1999;30(1):131-41. doi: 10.1016/ S0168-8278(99)80017-8.

10. Czaja AJ. Recurrent autoimmune hepatitis after liver transplantation: a disease continuum or a fresh start? Liver Transpl. 2009;15(10):1169-71. doi: 10.1002/lt.21809.

11. Hennes EM, Zeniya M, Czaja AJ, Parés A, Dalekos GN, Krawitt EL, et al. Simplified criteria for the diagnosis of autoimmune hepatitis. Hepatology. 2008;48(1):169-76. doi: 10.1002/hep.22322.

12. Díaz-Ramírez GS, Marín-Zuluaga JI, Donado-Gómez JH, Muñoz-Maya O, Santos-Sánchez Ó, Restrepo-Gutiérrez JC. Characterization of patients with autoimmune hepatitis at an university hospital in Medellín-Colombia: cohort study. Gastroenterol Hepatol. 2018;41(2):87-96. doi: 10.1016/j. gastrohep.2017.09.003.

13. Gautam M, Cheruvattath R, Balan V. Recurrence of autoimmune liver disease after liver transplantation: a systematic review. Liver Transpl. 2006;12(12):1813-24.

14. Montano-Loza AJ, Bhanji RA, Wasilenko S, Mason AL. Systematic review: recurrent autoimmune liver diseases after liver transplantation. Aliment Pharmacol Ther. 2017;45(4):485-500. doi: 10.1111/apt.13894.

15. Krishnamoorthy TL, Miezynska-Kurtycz J, Hodson J, Gunson BK, Neuberger J, Milkiewicz P, et al. Longterm corticosteroid use after liver transplantation for autoimmune hepatitis is safe and associated with a lower incidence of recurrent disease. Liver Transpl. 2016;22(1):34-41. doi: 10.1002/lt.24323.

16. Duclos-Vallée JC, Sebagh M, Rifai K, Johanet C, Ballot E, Guettier C, et al. A 10 year follow up study of patients transplanted for autoimmune hepatitis: histological recurrence precedes clinical and biochemical recurrence. Gut. 2003;52(6):893-7. https://doi.org/10.1136/gut.52.6.893.

17. González-Koch A, Czaja AJ, Carpenter HA, Roberts SK, Charlton MR, Porayko MK, et al. Recurrent autoimmune hepatitis after orthotopic liver transplantation. Liver Transpl. 2001;7(4):302-10. doi: 10.1053/jlts.2001.21449.

18. Narumi S, Hakamada K, Sasaki M, Freise CE, Stock PG, Roberts JP, et al. Liver transplantation for autoimmune hepatitis: rejection and recurrence. Transplant Proc. 1999;31(5):1955-6. doi: 10.1016/S0041-1345(99)00227-4.

19. Yao H, Michitaka K, Tokumoto Y, Murata Y, Mashiba T, Abe M, et al. Recurrence of autoimmune hepatitis after liver transplantation without elevation of alanine aminotransferase. World J Gastroenterol. 2007;13(10):1618-21. doi: 10.3748/wjg.v13.i10.1618. 